\title{
Use of radiobiology in medical jurisprudence, with particular reference to delays in diagnosis and therapeutic onset
}

\section{BLEDDYN JONES, MD SCD}

Gray Laboratory Oxford Institute for Radiation Oncology and Biology, University of Oxford, Old Road Campus Research Building, Roosevelt Drive, Oxford, UK

Address correspondence to: Professor Bleddyn Jones

E-mail: bleddyn.jones@oncology.ox.ac.uk

Objective: This paper considers aspects of radiobiology and cell and tissue kinetics applicable to legal disputations concerned with diagnostic and treatment onset delays.

Methods: Various models for tumour volume changes with time are reviewed for estimating volume ranges at earlier times, using ranges of kinetic parameters. Statistical cure probability methods, using Poisson statistics with allowances for parameter heterogeneity, are also described to estimate the significance of treatment delays, as well as biological effective dose (BED) estimations of radiation effectiveness.

Results: The use of growth curves, based on parameters in the literature but with extended ranges, can identify a window of earlier times when such tumour volumes would be amenable to a cure based on the literature for curability with stage (and dimensions). Also, where tumour dimensions are not available in a post-operative setting, higher cure probabilities can be achieved if treatment had been given at earlier times.

Conclusion: The use of radiobiological modelling can provide useful insights, with quantitative assessments of probable prior conditions and future outcomes, and thus be of assistance to a Court in deciding the most correct judgement.

Advances in knowledge: This study collates prior knowledge about aspects of radiobiology that can be useful in the accumulation of sufficient proof within medicolegal claims involving diagnostic and treatment days.

This paper describes some of the present radiobiological techniques, their scope and limitations, together with reasonable suggestions as to how numerical methods can sometimes be used to settle legal disputations mainly concerned with treatment delay issues arising in radiation oncology and more generally in oncology.

For some time, Law Courts have had to decide whether breaches of medical duties have occurred, and if so, whether such breaches have caused adverse clinical outcomes. In cancer therapy or surgery, e.g. delayed diagnosis (for whatever reason) is a typical complaint to be addressed. If the delay shown to be due to a breach of duty, it must then be proved, on the balance of probabilities, that the delay has caused an adverse outcome that probably would not otherwise have occurred. Expert radiologists are also required to assess imaging studies and comment on their technical and reporting qualities, as well as the dimensions at which a tumour may be found by imaging, but it is left to 
oncologists to determine the significance of any breach of duty found.

\section{METHODS}

Three methods of problem solving applicable to analyzing the impact of delays in diagnosis and therapeutic onset are considered:

\section{Use of cell and tumour kinetics}

Cell kinetics developed from the 1960s onwards. There are excellent but complex explanatory reference works by Cleaver, ${ }^{3}$ Steel, ${ }^{4}$ Tubiana, ${ }^{5}$ and Aherne et al. ${ }^{6}$ The essential features are as follows:

After the identification of the cell cycle, ${ }^{7}$ further concepts emerged from experiments which produced numerical parameter ranges found during growth of animal and human malignant tumours:

(1) cell cycle time $T_{C}$, (the time to traverse the cell cycle in which one cell becomes two daughter cells),

(2) growth fraction (GF) (the percentage of cells occupied in traversing the cell cycle),

(3) potential doubling time (the doubling time of a cell population given its $\mathrm{T}_{\mathrm{C}}$ and $\mathrm{GF}$, thus $\mathrm{T}_{\text {pot }}=\mathrm{T}_{\mathrm{C}} / \mathrm{GF}$ ).

The actual volume doubling time $\left(\mathrm{T}_{\mathrm{D}}\right)$ is normally considerably longer than the Tpot, due to the cell loss factor $(\phi)$, which quantifies the percentage loss from newborn cells as

$$
\varphi=\left(1-\frac{T_{p o t}}{T_{D}}\right) \%
$$

It follows that

$$
\mathrm{T}_{\mathrm{D}}=\mathrm{T}_{\mathrm{pot}} /(1-\phi)
$$

The cell loss factor is $100 \%$ in adult normal tissues, which allows each tissue to maintain a constant size at maturity. Lesser values occur in tumours and during normal maturation, which allows more rapid growth, but later increases in $\phi$ are followed by deceleration of growth ultimately to a steady-state condition of constant volume in normal tissues; increases in tumour $\phi$ produce longer volume doubling times in larger tumours, resulting in slower volume growth. Consequently, advanced tumours, most often in pre-terminal conditions, have very long doubling times, even though their potential doubling times remain short. ${ }^{8}$ This finding is a consequence of a restriction of tumour blood supply, increased interstitial fluid pressure, further pressure increases due to growth in confined volumes (both causes restrict the inward flow of blood) and progressive patient inanition causing nutrient deprivations that are essential for growth.

Many human tumour types have cell cycle times of 1-2 days, growth fractions of $10 \%$, leading to $\mathrm{T}_{\text {pot }}$ values of $10-20$ days, but with $\phi$ values often around $90 \%$, then volume doubling times can be between 100 and 200 days. Shorter times can occur, e.g. in squamous cell cancers where Tpot is around $4-5$ days, the $T_{D}$ is typically 40-70 days. Measured estimates of these parameters for many tumour histological classes are given in standard radiobiology textbooks, and are tabulated by Steel. ${ }^{4}$
Direct measurements of average tumour volume doubling times exist, but these values include the shorter and longer $T_{D}$ values that occur during the natural history of an individual tumour. Slower tumour growth with increasing volume occurs due to the above mentioned factors, including oxygen and nutrient deficiencies, which increases. Unfortunately, in clinical practice, the overall shape of a tumour volume growth curve may not be known if volumes have only been measured at two time points. It is then possible to estimate the average doubling time only; a third volume time point, or even more such points are required to estimate the degree of deceleration. Deceleration of growth results in sigmoidal (rather than an exponential growth curve) and can be fitted by the Gompertz equation, ${ }^{4}$ which can be used to fit growth curves in detailed animal studies, ${ }^{4}$ and also applicable to human tumours, ${ }^{4,9,10}$ although the monoexponential model fits the growth of developing lung metastases well. ${ }^{11}$ If there are only two available estimates of tumour volume, then a reasonable range of tumour volume doubling times that fall between the extreme limits of the longest and shortest reported times, or between a long doubling time (assuming to be 50 and 95\%) and double the Tpot value (if available). Examples of taking the published average volume doubling time, with a reasonable lower and upper limit, is sufficient to cover the phase space on a graphic which the sigmoidal growth curve can occupy, with also the advantage that even longer doubling times will inevitably lead to conclusions that a sufficiently large, detectable tumour would probably have been present at much earlier times.

Interested readers should consult the excellent review of cell kinetics by Tubiana, ${ }^{5}$ who also explained how the origins of metastatic tumours in breast cancer often occurred many years before mastectomy procedures.

If the $T_{D}$ values are not known in an individual patient, but the cell kinetic indices described above are known, then it is reasonable to use the above numerical relationships to predict the volume doubling time under typical cell loss factor conditions.

\section{Tumour volume expansion}

The increase in tumour volume with time can be represented in mathematical form by the following methods:

1. Linear growth: applicable to many slow growing human tumours

$$
V_{t}=V_{0} \cdot\left(1+K_{L} t\right)
$$

where $\mathrm{V}_{\mathrm{t}}, \mathrm{V}_{0}$ are volumes at time $\mathrm{t}$ and time 0 respectively, and $\mathrm{K}_{\mathrm{L}}$ the linear coefficient of growth (fractional increase per unit time). This is essentially a simplification of category two below when parameter A is very small (e.g. doubling times of several hundred days). The $\mathrm{K}_{\mathrm{L}}$ parameter is sometimes described as a "velocity", as in the "prostate specific antigen (PSA) velocity" used in prostate cancer marker studies. ${ }^{12}$ In linear (or steadystate) growth, the cellular doubling time is $1 / \mathrm{K}_{\mathrm{L}}$. Changes in PSA can be useful to assess tumour bulk with time, although the velocity parameter itself has no prognostic usefulness. ${ }^{13}$

(1) Exponential growth: 
(2) $\mathrm{V}_{\mathrm{t}}=\mathrm{V}_{0} \cdot \operatorname{Exp}[$ A.t $](1.4)$

(3) where $\mathrm{A}$ is the mono-exponential growth rate constant, which is $0.693 /$ [cellular doubling time].

(4) Gompertzian growth:

$$
\mathrm{V}_{\mathrm{t}}=\mathrm{V}_{0} \cdot \operatorname{Exp}[(\mathrm{A} / \mathrm{B}) \cdot(1-\operatorname{Exp}[-\mathrm{Bt}])]
$$

where $\mathrm{A}$ is now the exponential growth rate constant when the tumour is very small, B is a further parameter that controls the slowing down of growth in proportion to tumour volume. The volume doubling time (W) is $0.693 /$ A for very small tumours. Between two intervals of time, where the volumes change from $1 \mathrm{~cm}^{3}$ to any other larger value, it can be shown that:

(a) the cellular doubling time, itself related to the volume doubling time $w$, at any specified time is

(b) $\frac{0.693}{A} \operatorname{Exp}[B t](1.6)$

(c) the average doubling time $\left(w_{\mathrm{av}}\right)$ throughout a defined period of growth is obtained by integrating the previous expression and dividing by $t$ to give: (1.7)

(d) $0.693(\operatorname{Exp}[\mathrm{Bt}]-1) /(\mathrm{ABt})$

(e) the equivalent constant doubling time, $w_{\text {eq }}$, is

(f) $0.6936 \mathrm{Bt} /(\mathrm{A}(1-\operatorname{Exp}[\mathrm{Bt}]))(1.8)$

(g) and is obtained by solution of the condition where equation (1.7)=equation (1.8). This expression, when used within the standard exponential growth equation over the same interval of time, provides the same volume as the Gompertz growth curve, and can be used to provide a comparison with a Gompertzian fit, as shown in the results section.

(h) A linear approximation which covers the longer boundary of the Gompertz can be obtained using can be obtained using $w_{\mathrm{eq}}$ which is then used within the linear growth equation (1.3).

Plots of the three forms of $w$ are given in (Figure A1, Appendix A)

To estimate A and B it is necessary to have at least three or multiple data points, although assumptions can be made about the cellular volume at zero time, but the model assumes immediate and progressive slowing which may not naturally occur until a certain cell density is achieved and the further perturbations of an acquired vascular system and its inability to match tumour growth. If the minimum doubling time at a designated small tumour size $\left(\right.$ e.g. $\left.1 \mathrm{~cm}^{3}\right)$ is assumed, only two points are necessary. Obviously, the precision of the estimate will increase with the number of observed points, but in medicolegal situations there is usually only one or at two points. For example, a tumour may have been incorrectly undetected when at a volume of $\mathrm{V}_{0} \mathrm{~cm}^{3}$ (but is retrospectively found to be present) but is diagnosed at a future time $(\mathrm{t})$.

Then,

$$
\log _{e}\left[V_{t} / V_{0}\right]=[(A / B) \cdot(1-\operatorname{Exp}[-B t])]
$$

This function can be fitted to data using available mathematical software (e.g. Mathematica/Maple/Matlab), which will provide values for $\mathrm{A}$ and $\mathrm{B}$, and their confidence limits.
Alternatively, one can use a further function $\left(\mathrm{W}_{\mathrm{t}}\right)$ representing the change in cell numbers with elapsed time, as

$$
\mathrm{W}_{\mathrm{t}}=\log \left[\mathrm{N}_{\mathrm{t}}\right]-\log \left[\mathrm{N}_{(\mathrm{t}-1)}\right]
$$

Which is assumed to decay with time, governed by B, as in

$$
\mathrm{W}_{\mathrm{t}}=\mathrm{C} \cdot \operatorname{Exp}[-\mathrm{B} . \mathrm{t}]
$$

This is then transformed to

$$
\log \left[\mathrm{W}_{\mathrm{t}}\right]=\log \mathrm{C}-\mathrm{B} . \mathrm{t}
$$

and this function is plotted. The intercept and gradient B can then be found by a further fitting process such as linear regression, or manually. The assumption of simple exponential growth is reasonable if and only if a reasonable range of curve parameters are used to provide a likely phase space for tumour volume. These curves must be representative of a wide range of realistic growth rates (using growth rates that are slower and faster than the average quoted values), as in the examples below.

\section{Volume, diameter and radius}

Since the exponential formulations for tumour growth are based on cell number which are proportional to volume, appropriate conversions are required to transform three-dimensional volume growth into a linear scale for estimating linear dimensions of a tumour such as the radius or diameters of tumours.

Linear dimensions such as diameter, or radius, can be used in the same way as volume. For example, if the linear dimension is noted to be $L_{t}$ at time $t$ then the expression $\mathrm{L} t=\mathrm{L}_{0} \operatorname{Exp}[0.693 \mathrm{t} /$ $(3 w)$ ] can be used, where the volume doubling time, $w$ is multiplied by 3. The rationale is given in Appendix B. The use of more complex formulations for ellipsoidal shapes are probably unnecessary if only one linear dimension is used to measure maximum tumour diameter, as is the case in routine radiological reporting.

It is legitimate to reverse the arrow of time within deterministic growth equations, so that volumes or linear dimensions can be estimated at earlier times which are relevant to the problem being considered. Again, the earlier times during the natural history of a tumour's growth may involve shorter doubling times than in its later phases.

Some defence witnesses may argue that a tumour has developed within a relatively short time, consistent with clinical experience of complete remissions which regrow to become a large tumour within a few months; but such opinions should not hold for the entire natural history of a tumour, when at much earlier times a smaller number of cells would have been present. For example, it will take a tumour much longer to grow from say $10^{4}$ to be $10^{8}$ cells than it would from $10^{6}$ to $10^{8}$ cells. To discount such opinions, it is sometimes useful to estimate the age of a tumour, from one cell to become $10^{9}$ cells. The minimum tumour age $\left(\mathrm{t}_{0}\right)$ can be estimated from a simple rearrangement of the monoexponential growth equation, as: 


$$
t_{0}=\frac{\mathrm{w} \cdot \ln \left(10^{9}\right)}{0.693}
$$

where $w$ is the cellular doubling time.

In the example of ovarian cancer, which has a rapid growth rate (the measured average tumour cell doubling time is 33.3 days) ${ }^{4} 995$ days are required for such a tumour to develop. Even in the case of shorter doubling times $(15,20$, and 25 days), the time to develop a mass of $10^{9}$ cells (representing around $10 \mathrm{~g}$ of tissue) would be 448, 598 and 748 days respectively. For longer doubling times than the quoted average (which is based on only four observed cases of pulmonary metastases which probably have a faster growth rate than primary tumours), then time in excess of 748 days would be found. Consequently, it can be seen that durations far longer than a few months (as claimed by some witnesses) are necessary for the development of an observable tumour, even when the assumed growth rates are fast; it follows that slower growth rate parameters will take even longer times.

\section{Patterns of growth}

Tumour growth develops by centrifugal expansion, with limitations in some directions by tough fascial membranes, ligaments, bone etc. Tumours may also be attracted towards regions that have a better blood supply.

It is legitimate to reverse the arrow of time within deterministic growth equations, allowing volumes or linear dimensions to be estimated at earlier times.

\section{Estimates of tumour dimensions at earlier times}

To reverse the classical Gompertz equation in order to estimate tumour dimensions at earlier times denoted by $t$, the form of the equation becomes

$$
\mathrm{V}_{\mathrm{t}}=\mathrm{V}_{0} \cdot \operatorname{Exp}[(\mathrm{A} / \mathrm{B})] \cdot\left(1-\operatorname{Exp}\left[-\mathrm{B}\left(\mathrm{t}_{\mathrm{M}}-\mathrm{t}\right)\right]\right)
$$

where $t_{M}$ is the elapsed time for the tumour to grow between the two limits being considered.

\section{Tumour stage}

Civil law demands proof to the "balance of probabilities" level. In other words, the scientific probability must be above $50 \%$, e.g. a $51 \%$ scientific probability that a particular action has caused a deleterious effect.

Much emphasis is placed on tumour stage, and its relationship to prognosis from published series, although such statistics do not normally include the effect of significant treatment delays, and if so only in a small number of patients. And it is important to realize that although the stage remains the same for classification purposes after debulking surgery, the number of remaining cells to be eliminated by other forms of treatment has changed enormously, and this number will change with time. There may also be a greater number of residual cells after surgery if the tumour was larger than it would have been due to a delayed diagnosis. Tumour staging definitions sometimes change when new classifications are published: the latest version of the TNM or other international staging systems, such as FIGO in gynaecological cancer, should be consulted at the time of doing medicolegal work and confusion may arise when the treatment was given at a previous time before change in the classification. In situations of doubt, both systems (new and old) should be used.

Whereas stage does correlate with survival prospects it is not an infallible guide. Survival statistics presented with respect to stage often differ between publications and confidence limits may overlap. Staging systems are often designed for the convenience of the surgeon and are undoubtedly helpful in patient management. They do not allow for true tumour cell numbers or volume. For example, there is a huge latitude in the propensity for spread and for prognosis within what is called stage IB1 cervical cancer, ranging from a minimum depth exceeding $7 \mathrm{~mm}$ of invasion to a maximum diameter of $4 \mathrm{~cm}$. The risk of lymph node metastasis is mainly proportional to tumour size and of course the degree of invasiveness (a function of its biological potential and often related to tumour grade) so that a delay in diagnosis could be sufficient to allow a higher risk of metastasis (and a more unfavourable prognosis) despite the stage being the same. The smallest tumours in this category would be expected to have a $10 \%$ lymph node metastatic yield, but the largest around 30\%. In one personal series, the Stage IV patient survival results were better than those in Stage IIIB, due to there being smaller tumour bulk in many of the patients in the former case. ${ }^{14}$ Thus, the staging system is not sufficiently sensitive. This problem recalls the views of Aristotle that biology was so variable that perfect categorization is impossible. ${ }^{15}$ It follows that if stage has not changed but the tumour volume has substantially increased, it is then essential to consider scientific probabilities of tumour control, as discussed above, where the individual patients prospects for cure are assessed at two different time points dependent on the relevant diagnostic delay time, using a reasonable but wide choice of TCP values in order to obtain the total cell kill parameter $(L C)$.

This becomes especially important where there is known microscopical (or residual) tumour after surgery: shorter doubling times must then be assumed, rather than the longer average times used in the case of larger well-established tumours.

\section{Lack of growth or regression}

Whereas benign and malignant tumour grow with time, other medical conditions may be static and show no volumetric change many years after irradiation. If a diagnosis of cancer cannot be made using this criterion, it can sometimes lead to an alternative diagnoses, e.g. old but volumetrically stable calcified ring-like lesions from previous tuberculosis, toxoplasmosis or cysticercosis, depending on the medical history, radiological features and results of other investigations. Other conditions such as schistosomiasis, syphilis and sarcoidosis may also simulate the appearance of a malignant tumour.

\section{Surrogates of tumour growth: tumour markers}

Quantitative assays of molecular tumour markers, which are usually proteins produced by the tumour cells, can be used to assess changes in the tumour population with time. This requires 
an assumption that tumour cells are producing roughly equal amounts of the protein, which changes with time in accordance with linear or exponential kinetics. Examples are the PSA and CA125 antigen in ovarian cancer. These methods are not infallible, since

(1) normal tissues can produce elevated levels in pathological conditions and there is a lower normal range boundary beneath which the tumour cannot be detected.

(2) The assay range may have restrictions at a high levels.

(3) Further tumour cell mutations may result in reduced tumour marker production.

(4) The clearance of tumour markers from the circulation may be reduced due to subtle or marked changes in kidney or liver function due to increasing age of other pathologies.

However, they are useful in relatively well-differentiated, highgrade cancers when the tumour cell burden is moderate, i.e. in relatively small- or medium-sized tumours.

(1) Use of Poisson Statistics for Cure Probabilities The Poisson probability theory of Simon Denis Poisson (1781-1840) was published in 1837 as Recherches sur la probabilité des jugements en matière criminelle et en matière civile ("Research on the Probability of Judgments in Criminal and Civil Matters"). ${ }^{16}$ It considered probabilities associated with wrongful criminal convictions, arguably the first example of applied mathematics to society and is now extensively used in science and industry.

Poisson deftly simplified the Binomial formulation for conditions where the numbers of trials were large, but the chances of success small. The application in cure statistics was emphasized by Porter (a radiotherapist). ${ }^{17}$ Since the probability of no successful outcomes ( $r$ ) is given by

$$
P_{[r=0]}=e^{-m}
$$

for an expected mean outcome of $m$, then if say three "lethal injuries" are expected (on average) in a cell following treatment (so $m=3$ ), then the probability of no lethal injury is $e^{-3}=0.05$ (that is $5 \%$ ). This probability is then called the surviving fraction (SF). It follows that if $\mathrm{N}$ such cells were present at the start of treatment then the surviving fraction of cells will be $N \times \mathrm{SF}=0.05 \mathrm{~N}$. So, if the cell number was originally 100 , only 5 survive. A further Poisson statistical procedure is then necessary to estimate the tumour cure (or control) probability (TCP). It is assumed that TCP $=1$ (or 100\%) when no living tumour cells remain. Using the Poisson formula again, the probability of no remaining cells is given by $P_{[r=0]}=e^{-m}$, where $m$ is now the average number of surviving cells (given by $N \times \mathrm{SF}$ above). After treatment, if an expected average of 5 cells remain, the TCP is given by $e^{-5}$ which is 0.007 . If the cell killing was more efficient and the expected average number of remaining cells of one, then $\mathrm{TCP}=e^{-1}=0.37$ (or $\left.37 \%\right)$.

The complete formulation is then

$$
T C P=e^{-N e^{-L E}}
$$

where $L E$ are the expected lethal events which are related to radiation or drug dosage etc. using separate equations and $\mathrm{SF}=N e^{-L E} \cdot(2.3)$

The resulting Poisson family of radiation dose-response curves are steep since they apply to a single individual, where it is assumed that all cells are equally sensitive to treatment. When applied to populations of different individual tumours in different patients they become shallower and consistent with clinical results obtained from human populations. This effect is due to heterogeneity of the radiosensitivity parameters that control cell killing with dose. For individual tumours, the Poisson curves are probably closer to reality for smaller tumours or where there is a microscopical burden of tumour cells to be eradicated, but even then heterogeneity needs to be included. This general principle is shown with further equations and graphically in (Figure C1, Appendix C), where the steep individual curves are based on seven different $\alpha$ radiosensitivity parameters but the overall averaged probability outcome is that of a shallower curve for the same population of seven different individuals. Some population healthcare modelling studies have used considerable heterogeneity in the assessment of delays in starting radiotherapy or combined chemoradiotherapy, predicting only small detriments in tumour control for relatively long treatment delays, ${ }^{18}$ depending on the tumour subclass. Such conclusions cannot apply to individual patients, and especially in post-operative situations where there are fewer cells present and the dose-response curves are probably steeper. Then, cell number increases due to the delays can become more significant.

This can be seen from computer simulations that use conventional Poisson Statistics to estimate the scientific probability ( $\mathrm{P}$ of between 0 and 1 ) of cure (elimination of all cells), where for treatment, say 6 months earlier the number of cells is reduced by the exponential formula for assumed doubling times of 30-120 days. Treatment given at earlier times $(\mathrm{t}$ days earlier) can demonstrate the potential deleterious effect of treatment delays. In such estimations, the distinction between individual tumours and a population of tumours must be made, since the heterogeneity of radiosensitivity and other parameters can reduce the slope of dose response curves (see Appendix C). Heterogeneity can be introduced by computer random sampling techniques or by inclusion of standard deviations in analytical equations, or by assuming different outcome probabilities from the same treatment schedule.

Another approach is useful in special circumstances, e.g. where a treatment has been given unsuccessfully to eradicate a microscopical tumour burden after a delay of say 6 months. The number of tumour cells and their radiosensitivity parameters cannot be known with precision. To overcome these uncertainties, it is assumed that $N=10^{3}, 10^{4}$, $10^{5}, 10^{6}$ cells were present with respective TCP values of 0.4 , $0.2,0.1$ and 0.01 , which would, on the balance of probabilities not result in cure, since they are all predicting less than $50 \%$ statistical probability of cure. A conservative number of $10^{9}$ cells is normally taken to represent $10 \mathrm{~g}$ or $10 \mathrm{~cm}^{3}$ clinically detectable volume of tumour. ${ }^{19}$ It is then possible to use the same the overall SF for the particular treatment given 
(from equation 2.3 above) and apply this again at a time $t$ days earlier when the smaller cell number is

$$
N_{t}=N e^{-\frac{0.693 . t}{w}}
$$

Note that a negative exponential function is used to estimate $\mathrm{N}$ at earlier time. Appendix $\mathrm{C}$ provides the equations for the TCP at the two different times of interest.

This method has a considerable advantage in that all the major treatment related parameters that influence outcomes are implicitly included within parameter $L E$ (see Equation $\mathrm{C} 1$ in appendix $\mathrm{C}$ - which includes cellular radiosensitivities (and so the $\alpha / \beta$ ratios), cell division rates and any other factor contributing to successful radiotherapy) will be the same for identical treatments initiated at each of the two different time points. Further details of the $L E$ parameter is given in appendix C.

In this way considerable parameter heterogeneity has been introduced into the separate Poissonian estimates.

(2) Biological effective dose (BED).

This elegant concept has led to many practical applications by means of numerical assessments of radiation effectiveness. ${ }^{1,2,20}$

The formulation for standard megavoltage radiation the basic expression for BED is:

$$
B E D=n d\left(1+\frac{d}{\frac{\alpha}{\beta}}\right)
$$

For any specified $\mathrm{BED}$, the equivalent dose in $2 \mathrm{~Gy}$ fractions (the EQD-2) is simply:

$$
\mathrm{EQD}_{2}=\mathrm{BED} /(1+2 /(\alpha / \beta))
$$

BED is an end point specific concept and individual values is therefore usually written in the form $\mathrm{Gy}_{[x]}$, where $x$ is the $\alpha / \beta$ ratio used in the BED calculation.

The standard equation can be modified to account for different requirements, as in:

$$
B E D=n d\left(A+B \frac{d}{\beta}\right),
$$

Where A and B are separate functions of changing radiation quality, e.g. when using protons or heavier ions, ${ }^{20}$ and $\mathrm{B}$ alone may change with radiation dose rate or if inter fraction intervals are short. ${ }^{21}$

Since radiation effects in tissues are non-linear with dose, the use of dose itself may not correlate with bioeffectiveness, since the latter is also influenced by fractionation of the treatment, dose rate, radiation quality etc.

Isoeffective dose-fractionation calculations and by implication identification of dose fractionation schedules that are not isoeffective.

- unintended treatment interruptions,
- errors in treatment delivery,

- dose rate and dose-fractionation changes,

- technique comparisons

- re-treatments,

- clinical trial design,

- equivalent chemotherapy dosage,

- different radiation qualities (e.g. protons and other charged particles).

$\mathrm{BED}$ is defined as the total dose required to achieve a specified effect if that dose is given in very small fractional sizes. ${ }^{1}$ It is possible to estimate isoeffective doses and compare different dose fractionation schedules and the potential effects of overand underdosage, the influence of dose rate changes and changes with in bioeffectiveness with time. There are some pitfalls, such as that the $\alpha / \beta$ ratio must be known and that a single BED value cannot represent an entire treatment (unless the dose is uniform), so separate BEDs must be used where dose varies within an individual treatment. The $\alpha / \beta$ ratio may sometimes be varied to search for robust conclusions.

In general, BED will correlate with normal tissue effects and tumour control probabilities and, although there will be threshold BED to achieve certain classes of tissue effects and will at higher BED produce no additional effects; the dose-response relationship for tumour control will also depend on an effective threshold BED and similarly reach saturation level near a probability of 1 . It is often assumed as a rule of thumb that a $1 \%$ increase in BED beyond the saturation value will be associated with (at least) a 1-2\% increase in normal tissue side-effects.

The relationship between dose, BED and volume of an organ irradiated is complex. At the present time, each of the large numbers of models that estimate volume-related effects are not regarded as highly accurate, although in the case of a single patient-if the prediction show large differences in outcomes-they can be a useful guide. The simpler use of good representative BED values at points of interest that are likely to influence the tissue response is probably sufficient evidence of under- or overdosage. These should be selected carefully under the guidance of an experienced clinician.

Worked examples of BED calculations are available elsewhere $e^{1,2}$ as are ranges of $\alpha / \beta$ ratios. It should be noted that $\alpha / \beta$ ratios are well established for late normal tissue side-effects (2 Gy for brain and spinal cord; $3 \mathrm{~Gy}$ for lung and other soft tissues). For tumours, a greater range exists and for tumour control the parameter should be varied to test any assumption made. It is also the case that isoeffect calculations show little change when $\alpha / \beta$ of $7 \mathrm{~Gy}$ and above are used.

\section{RESULTS}

\section{Volumetric and diameter studies}

First, it is instructive to consider the growth of a tumour from an initial volume of $1 \mathrm{~cm}^{3}$ to become $100 \mathrm{~cm}^{3}$ at different times depending on the growth rate. Figure 1 shows the growth curves for four different mono-exponential doubling times (40, 80 and 120 and 180 days) which pass between the same initial and final conditions, but with all curves diverging from the initial condition. Also shown is a Gompertz-type curve, based on an initial doubling time of 40 days at a volume of $1 \mathrm{~cm}^{3}$, which can be 
Figure 1. Four examples of exponential tumour growth with increasing time for volume doubling times of 60-180 days, and one Gompertz(sigmoidal) growth curve which matches the volume of the 180 doubling time example at just over 3 years

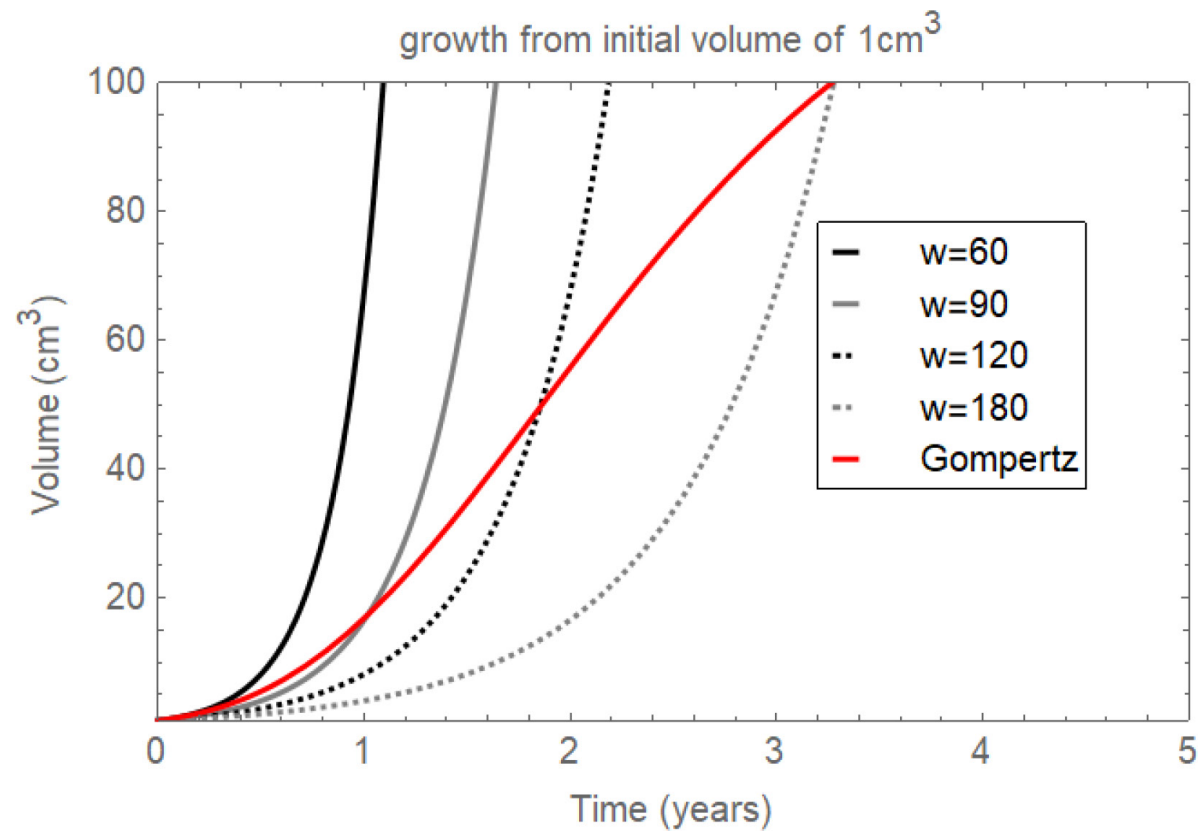

expected in a small squamous cell carcinoma. It can be seen that only the 180-day doubling time corresponds with the Gompertz curve at both the lower and upper limits of volume displayed. The Gompertz volumes fall within the area of the graph (or phase space) bounded by the other doubling times and at very early times, it is closest to the shortest doubling time curve. In a real situation of two volumes being known (e.g. a missed diagnosis at $1 \mathrm{~cm}^{3}$, but confirmed retrospectively, and that of a large $100 \mathrm{~cm}^{3}$ cancer 3.27 years later), then the longest doubling time or the Gompertz can be used.

Although it is possible to estimate the tumour volumes at times before 3.27 years from Figure 1, it can be confusing to do so. The alternative is to estimate the volume at earlier times by reversing the direction of time as shown in Figure 2, where the curves diverge from the initial condition of the large $100 \mathrm{~cm}^{3}$ volume to

Figure 2. Time is now reversed, but using the same doubling times and Gompertz parameters, for volumes at earlier times than a reference volume of $100 \mathrm{~cm}^{3}$

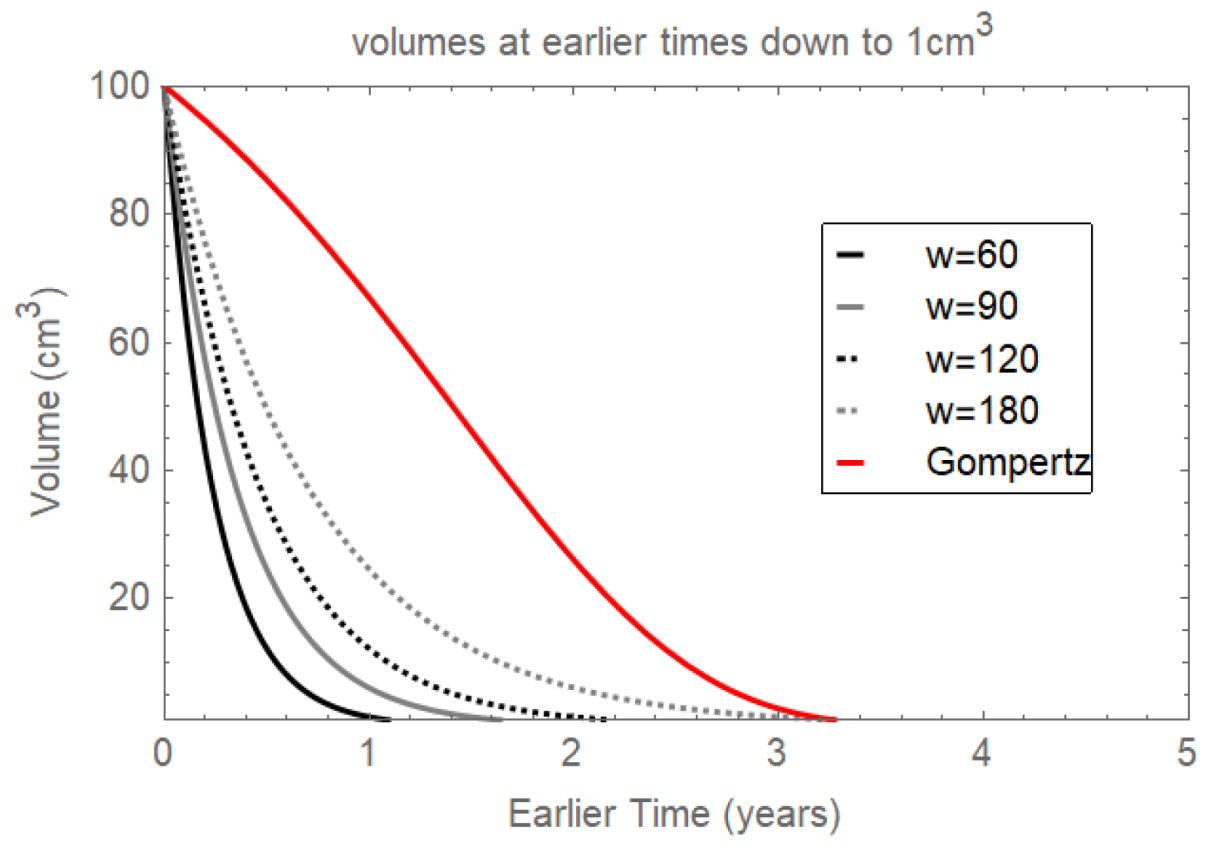


Figure 3. Tumour diameter at earlier times for respective assumed volume doubling times, with a linear approximation of a Gompertz curve trio provide a further boundary. Longer doubling times than shown here would predict even larger tumour diameters at earlier times

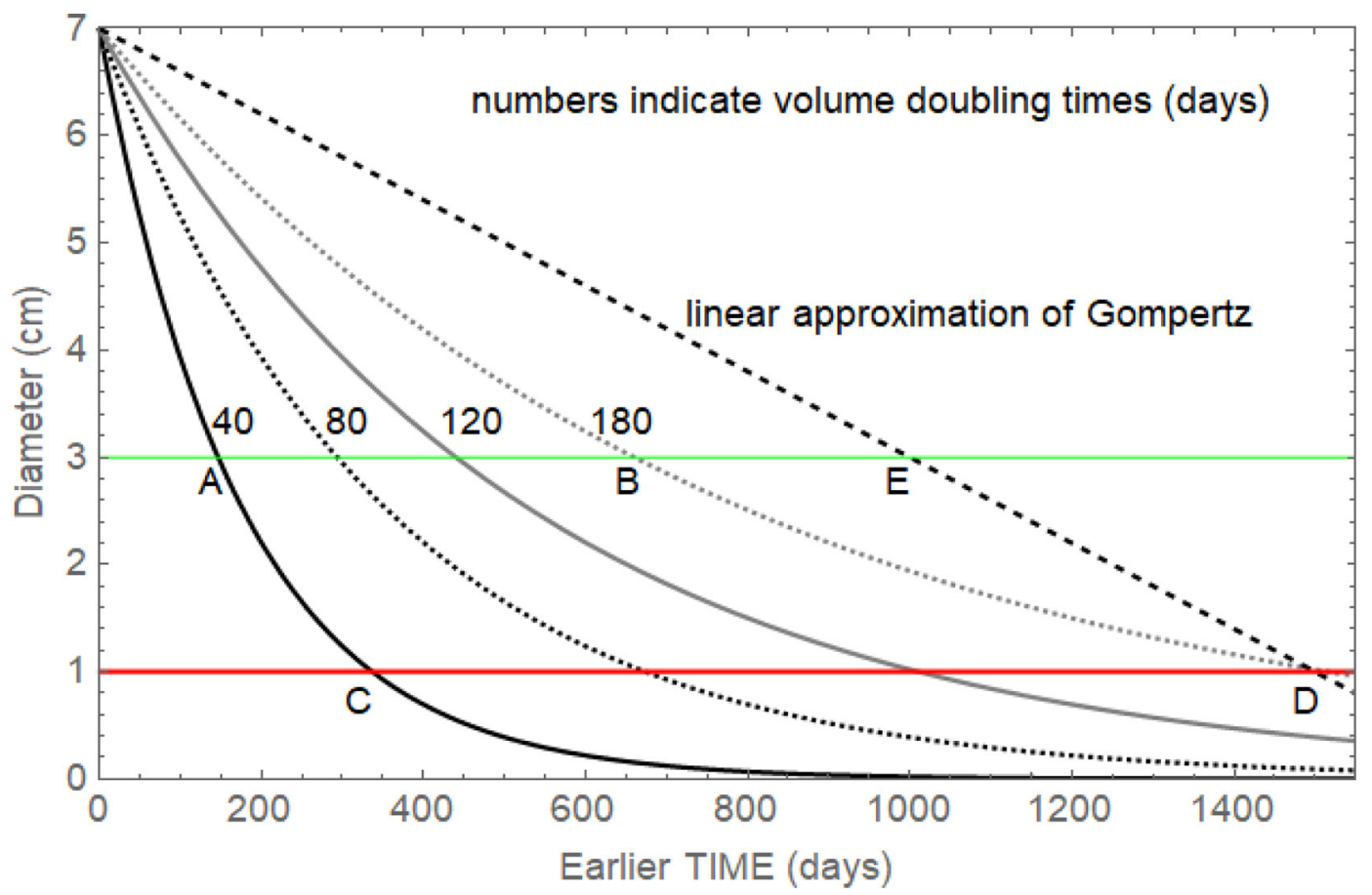

give quite different estimates of tumour volumes at earlier times. Such a plot has the advantage of showing that the Gompertz occupies a phase space which signifies larger tumour volumes than found in any of the other monoexponential curves. This has some important implications, since the use of the monoexponential doubling times provide a smaller tumour volume that is more difficult to detect. Thus use of several monoexponential curves provide a worse-case scenario, and if the volume is in a detectable range for a diagnostic technique, then it is highly likely to be detected if it is actually larger. When matters of curability (which is usually inversely related to tumour volume) are considered, the Gompertz curve indicates a larger volume at prior times, which would be the best choice; the alternative would be to provide a severe constraint on tumour volume for curability when using the monoexponential with longer doubling times than the mean value in the literature (as represented by the 120 and 180 day doubling times compared with an assumed mean of 80 days in Figures 1 and 2), which is an entirely reasonable assumption for most squamous cell cancers.

\section{A clinical example}

A patient with a $7 \mathrm{~cm}$ diameter metastatic cervical cancer tumour at the time of diagnosis but where competent diagnosis could be achieved at diameters of say $1-3 \mathrm{~cm}$ using various methods (clinical examination or imaging) at previous times. In such a case, possible diameters at earlier times are plotted using four different volume doubling times (short, mean, and long) which represent reasonable limits of probable growth rates (Figure 3). These volume doubling times are trebled within these estimates to convert from volume to linear growth. The red line indicates the probable diagnostic scan detection size in the anatomical site of interest, and the green line the size of tumour beyond which cure would be more difficult to obtain. The window of opportunity for a high chance of tumour eradication (curative treatment) for successful treatment would be defined by the area bounded by the intersections of the outer two curves with the straight lines, and especially so the "middle ground" between 400 and 600 days earlier, which corresponds to around 14-22 months beforehand. Since this interval exceeds 6 months, it follows that 6 monthly diagnostic scans would be sufficient to achieve effective follow-up. It is not possible to superimpose a Gompertz curve in this example, since only the initial condition is known, but from the previous example in Figure 2, such a curve would occupy a phase space above the other curves, that is have larger volumes at the same times. One possible way of overcoming this problem would be to construct a line which meets the longest doubling time curve at the required smallest detectable size $(1 \mathrm{~cm}$ diameter is assumed here), since the Gompertz curve is pseudolinear for a considerable duration, as seen in Figure 3. This describes the approximate largest diameters at prior times for the class of tumour with these representative doubling times. The shortest doubling time curve (40 days) provides the worst case for rapid growth, the mean of 80 days and the further longer times of 120 and 180 days represent slower growth and are all bounded within rectangle $\mathrm{ABDC}$, but also within triangle BED for the worse case of slowest growth given by the linear approximation to the Gompertz (which is easier to plot). The total window of opportunity to detect the cancer while at a curable size (assumed to be $3 \mathrm{~cm}$ in diameter as a relatively modest assumption) is then given by the rectangle AEDC. The prospects for earlier detection of the cancer by clinical examination or imaging (depending on the 
Table 1. Treatment is given six months earlier than was actually the case

\begin{tabular}{|l|l|l|l|l|l|}
\hline $\begin{array}{l}\text { Initial } \mathrm{P}(\%) \\
\mathbf{6} \text { months earlier }\end{array}$ & $\mathrm{TD}=\mathbf{3 0}$ days & $\mathrm{TD}=\mathbf{4 0}$ days & $\mathrm{TD}=\mathbf{6 0}$ days & $\mathrm{TD}=\mathbf{8 0}$ days & TD = 120 days \\
\hline $1 \%\left(10^{7}\right)$ & $93 \%$ & $82 \%$ & $56 \%$ & $38 \%$ & $20 \%$ \\
\hline $10 \%\left(10^{6}\right)$ & $96 \%$ & $90 \%$ & $75 \%$ & $62 \%$ & $44 \%$ \\
\hline $30 \%\left(10^{5}\right)$ & $98 \%$ & $95 \%$ & $86 \%$ & $78 \%$ & $65 \%$ \\
\hline $40 \%\left(10^{4}\right)$ & $99 \%$ & $96 \%$ & $89 \%$ & $82 \%$ & $72 \%$ \\
\hline
\end{tabular}

Summary statistics: mean $=75.8 \%$; $95 \%$ confidence limits of mean: $66.1-85.5 \%$; median $82 \%$.

Statisticaltumour cure probabilities (from 0 to 1,but converted to percentages) for different assumed numbers of cells at thestart of chemoradiotherapy (shown in parentheses in the first column), whichgive rise to different cure probabilities shown in the first column for the treatment which failed. The variation in the cellular doubling times are shown.The results are the expected cure (total cell elimination) percentages in thematrix if treatment was delivered 6 monthsearlier. There would be higher percentage cure estimates if treatment had been commencedat any earlier time. It should be noted that the shorter range of doublingtimes are more likely to occur in this situation, down to a 1 month doublingtime in cases of microscopical tumour burdens after surgery.

body location) can then be investigated using typical intervals of time for good follow-up practices, e.g. 3 monthly examinations or 6 monthly or annual imaging studies.

\section{Tumour cure probabilities}

For individual tumours, the Poisson distribution can be used by assuming distinct probability levels, which should be less than 0.5 for instances where cure was not actually achieved. The results in Table 1 are obtained for the same treatment given 6 months earlier (using equation 2.4). In most instances, there are considerable gains in TCP to over 50\%. It is important to realize that since the baseline TCP values are less than $50 \%$, the radiosensitivities and other factors that determine cure will be unfavourable, thus all forms of tumour cell resistance are included within the parameter $L E$ used in the above equations. The delay times neglect a short delay between surgery and the first possible time to start treatment, which would be around 2 weeks.

The 6 month earlier treatment estimates given in Table 1, from a low expectation of cure of below $50 \%$ (range 1-40\%), show that in most instances, the expectation of cellular elimination (cure) is well above $50 \%$ as a consequence of reduced cell numbers if treatment had been delivered 6 months earlier. This appears to be a very robust finding given the wide range of expected cure rates below 50\% used. The summary statistics shows that the $95 \%$ confidence limits are above $50 \%$. Thus, when using this method and these parameter ranges, on the balance of probabilities the treatment probably would have been far more effective if given 6 months earlier, especially when the shorter doubling times are considered and which are more likely to occur for microscopical residual disease in the case of relatively fast growing tumours such as squamous cell cancers. The same conclusion cannot be made for treatment given 3 months earlier, as shown in Table 2, and where the gain in cure probability estimates are not so high as in Table 1, and the lower 95\% confidence limit falls below $50 \%$. A table for treatment given 4 months earlier is not provided, but the estimated mean cure probability is $62.9 \%$ (95\% CL: 52.1-73.7\%), the lower limit being just above 50\%, so may be regarded as significant. This corresponds well with the UK NHS recommendation that cancer treatment should begin within 62 days of referral. This limit is close to the average volume doubling time of many common cancers such as the squamous cell type.

The same principles can be shown in graphical form, but with assumptions made about radiosensitivities, as in Figure 4 for an individual tumour and in Figure 5 for a population of tumours. The results are shown with the option of total dose variation which estimates the additional dose required to compensate for treatment delays, although in practice dose escalation will produce additional tissue complications. Figure 4 shows that the standard dose in this situation (45 Gy in 25 treatments) provides a TCP of around $20 \%$, which is reasonable for a treatment which actually failed. The TCP values become greater than $50 \%$ for treatments starting around 90 days earlier and for all earlier times beyond 90 days.

In the case of a population of tumours, each with different characteristics in terms of doubling times and radiosensitivites, Figure 5 shows less marked increments in TCP changes for earlier treatments, and the standard dose of $45 \mathrm{~Gy}$ produces a very low TCP. Even so, the average TCP rises to over 50\% if the

Table 2. As in Table 1 but for treatment given 3 months earlier than was actually the case

\begin{tabular}{|l|l|l|l|l|l|}
\hline $\begin{array}{l}\text { Initial P(\%) } \\
\mathbf{6} \text { months earlier }\end{array}$ & $\mathrm{TD}=\mathbf{3 0}$ days & $\mathrm{TD}=\mathbf{4 0}$ days & $\mathrm{TD}=\mathbf{6 0}$ days & $\mathrm{TD}=\mathbf{8 0}$ days & TD = 120 days \\
\hline $1 \%\left(10^{7}\right)$ & $56 \%$ & $38 \%$ & $20 \%$ & $12 \%$ & $6 \%$ \\
\hline $10 \%\left(10^{6}\right)$ & $75 \%$ & $62 \%$ & $44 \%$ & $35 \%$ & $25 \%$ \\
\hline $30 \%\left(10^{5}\right)$ & $86 \%$ & $78 \%$ & $65 \%$ & $58 \%$ & $49 \%$ \\
\hline $40 \%\left(10^{4}\right)$ & $89 \%$ & $82 \%$ & $72 \%$ & $66 \%$ & $58 \%$ \\
\hline
\end{tabular}

Summary statistics: mean $=52.9 \%$; $95 \%$ confidence limits of mean: $43.2-62.2 \%$; median $58 \%$. 
Figure 4. The effect of improving tumour cure probability for earlier treatment onset times for an individual tumour that would otherwise follow the black dose response curve for the actual treatment start time. Radiobiological characteristics: $\alpha=0.25 \mathrm{~Gy}{ }^{-1}$, $b=0.025 \mathrm{~Gy}^{-2}$, cellular population doubling time $=60$ days, $5 \times 10^{5}$ cells present when actual treatment started

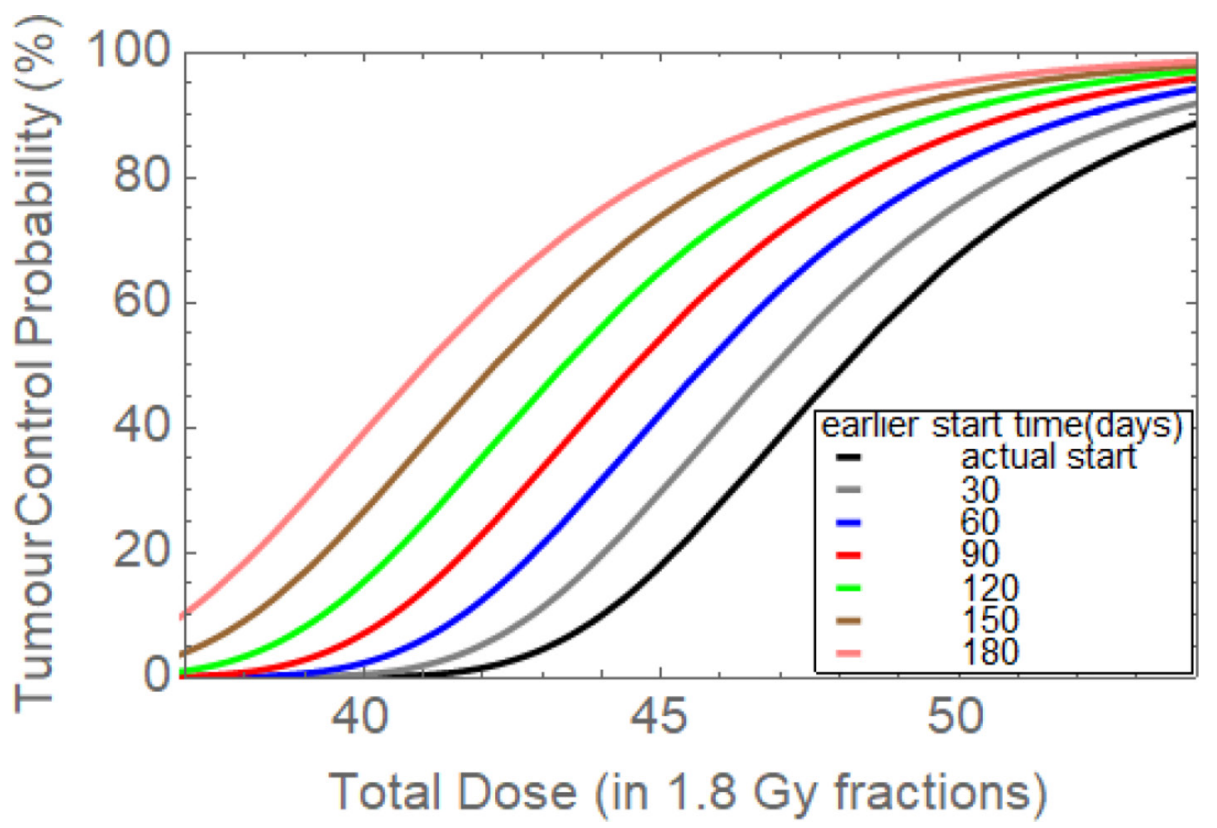

same dose is given 90 days earlier or at all earlier times beyond 90 days.

\section{DISCUSSION}

The numerical methods shown above are now standard in scientific research but need careful adaptation for legal use. Testing a hypothesis by using a wide range of possible parameters can overcome inherent uncertainties in some specific situations: a robust result can demonstrate that a probable outcome either can or cannot be achieved. Should robust modelling using a wide range of parameter variation lead to a conclusion that a window of opportunity exists within a range of time, this should be taken seriously if accompanied by curative treatments being available within such times.

Figure 5. The effect of improving tumour cure probability for earlier treatment onset times for a population of 100 patients which would follow the black dose response curve for the actual treatment start time. Radiobiological characteristics: mean $\alpha=$ $0.25 \mathrm{~Gy}^{-1}(\sigma=0.025), b=0.03 \mathrm{~Gy}^{-2}$, cellular population doubling time mean $=60$ days $(\sigma=1.25$ days, log-normal distribution), $5 \times$ $10^{5}$ cells present when actual treatment started

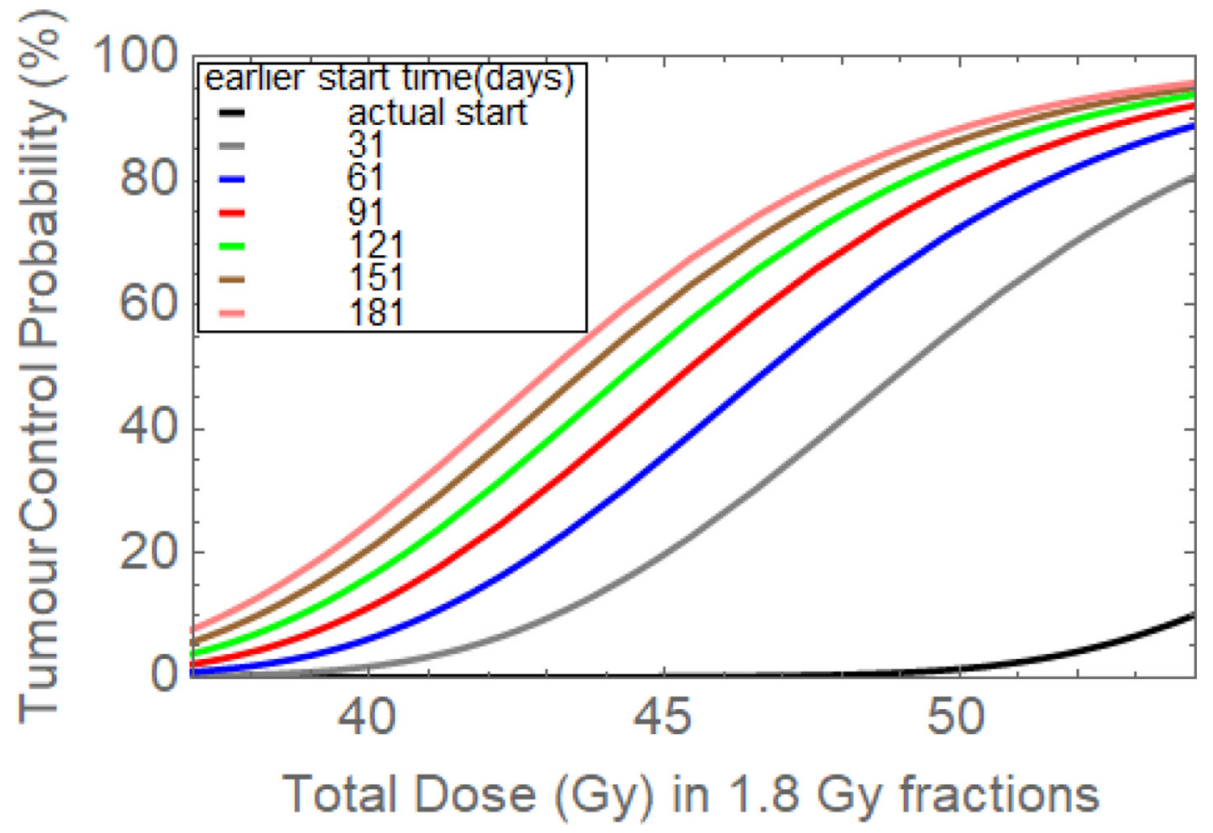


The balance of probabilities concept in Civil Law is often restricting, and sometimes frustrating to the medical scientist, as it can be argued that a change in the statistical probability of cure from $51 \%$ or greater down to $49 \%$ would be sufficient to establish causation, but a reduction in statistical cure probability from 35 to $10 \%$ due to some malpractice may not be sufficient proof to the court, a situation which can be frustrating to medical observers. Perhaps, a new definition of change may be necessary in the future (e.g. a reduction in cure prospects by $50 \%$ or more may be more satisfactory but even such a definition is open to criticism, for example if a risk of $2 \%$ is reduced to $0.05 \%$ ).

It is sometimes the case that serial observations of tumour volume has been possible and analyzed using the Gompertz equation to provide doubling times. For example, the report of Finlay et $\mathrm{al}^{10}$ has been used extensively to provide guidance as to the natural history of liver metastases from colorectal cancer, allowing informed decisions to be made regarding the time at which removal of the primary cancer would have prevented such developments. Such guidance may show that claims about delayed diagnosis have not resulted in failure to cure. Further advice is given by Friberg et al elsewhere. ${ }^{22}$

These precepts are a guide for those doing expert witness work, but it cannot be certain that they would be accepted in uniform manner in every Law Court where so much depends on presentation and interpretation.

There is no scope within the present paper to extend the arguments made here to detailed study of errors that occur during radiotherapy delivery, as described in other reports. ${ }^{23-25}$ Indeed, there is a need to formalize what can be done in such situations to establish error and its consequences. For example, the problem of geographic tumour miss is amenable to BED and TCP estimates ${ }^{26}$ even in particle therapy. Overdosage is easier to assess, but under dosage requires more complex TCP and BED estimates, and geographical miss may reduce TCP but increase normal tissue late effects. These can be related to known dose-response curves, their slopes (or $\gamma$ factors) with use of data in QUANTEC publications. ${ }^{27}$ Another issue is whether appropriate radiobiological corrections have been made to known errors detected during radiotherapy, since errors may be compounded if incorrect compensations are applied. A recent Royal College of Radiologists report, concerned with well-established methods for compensating delays within a treatment course, has highlighted the need for formal training in these areas ${ }^{28}$ since the existing training structures in Clinical Oncology and Medical Physics do not provide adequate opportunities to solve such problems with confidence. Perhaps, the British Institute of Radiology could commission helpful publications and foster further discussions in this important aspect of radiotherapy as well as in the assessment of legal disputations.

The literature concerning the treatment delays is imperfect. There are articles which discuss the causes of delays, from professional reasons to the techniques used, ${ }^{29,30}$ with indications of deleterious long-term outcomes in some cancers especially in the head and neck region ${ }^{31,32}$ where rapidly growing squamous cell cancers predominate. It is not a topic amenable to the design of a randomized study for obvious ethical reasons, so the literature is retrospective and uncontrolled with many confounding factors. There are even some "perverse" results, as in small cell lung cancer where prompt treatment is associated with a worse prognosis, probably due to the effects of over intensive therapy in an incurable condition. ${ }^{33}$

Experience in the subject of cell kinetics and radiation modelling is diminishing with time; new researchers are concentrating on molecular mechanisms, epidemiology, or clinical trial based research. Consequently, it is important that the basics of this subject is set down for future reference. Also, there is a need for an established reference which both claimant and defendant advisors can study in order to provide appropriate advice, rather than-in some instances-rely on poorly informed statements or over critical views about the role of mathematics in medicine, which may lead to misjudgements.

\section{REFERENCES}

1. Jones B, Dale RG, Deehan C, Hopkins KI, Morgan DA. The role of biologically effective dose (bed) in clinical oncology. Clin Oncol 2001; 13: 71-81. doi: https://doi.org/10.1053/ clon.2001.9221

2. Dale RG, Jones B. Radiobiological modelling in radiation oncology. British Institute of Radiology 2007; 2016.

3. Cleaver JE. Thymidine Metabolism and Cell Kinetics. Amsterdam: North-Holland; 1967.

4. Steel GG. p5-55 in Growth Kinetics of Tumours. Oxford: Clarendon Press; 1977.

5. Tubiana M. The kinetics of tumour cell proliferation and radiotherapy. Br J Radiol 1971; 44: 325-47. doi: https://doi.org/10. 1259/0007-1285-44-521-325
6. Aherne WA, Camplejohn RS, Wright NA. Introduction to Cell Population Kinetics. London: Edward Arnold; 1977.

7. PELC SR, HOWARD A. Techniques of autoradiography and the application of the STRIPPING-FILM method to problems of nuclear metabolism. $\mathrm{Br}$ Med Bull 1951; 8(2-3): 132-5. doi: https:// doi.org/10.1093/oxfordjournals.bmb. a074095

8. Bolger BS, Cooke TG, Symonds RP, MacLean AB, Stanton PD. Measurement of cell kinetics in cervical tumours using bromodeoxyuridine. Br J Cancer 1993; 68: 166-71. doi: https://doi.org/10.1038/bjc. 1993.307
9. Geddes DM. The natural history of lung cancer: a review based on rates of tumour growth. Br J Dis Chest 1979; 73: 1-17. doi: https://doi.org/10.1016/0007-0971(79)900020

10. Finlay IG, Meek D, Brunton F, McArdle CS. Growth rate of hepatic metastases in colorectal carcinoma. Br J Surg 1988; 75 : 641-4. doi: https://doi.org/10.1002/bjs. 1800750707

11. Trott KR, Heinze HG, Kiesel B, Kollmann G, Kummermehr J. Growth rate of pulmonary metastases. Strahlentherapie 1979; 155 : 748-53.

12. Carter HB, Pearson JD, Metter EJ, Brant LJ, Chan DW, Andres R, et al. Longitudinal 
evaluation of prostate-specific antigen levels in men with and without prostate disease. JAMA 1992; 267: 2215-20. doi: https://doi. org/10.1001/jama.1992.03480160073037

13. Vickers AJ, Thompson IM, Klein E, Carroll PR, Scardino PT. A commentary on PSA velocity and doubling time for clinical decisions in prostate cancer. Urology 2014; 83: 592-8. doi: https://doi.org/10.1016/j. urology.2013.09.075

16. https://www-history.mcs.st-andrews.ac.uk/ Biographies/Poisson.html

17. Porter EH. The statistics of dose/cure relationships for irradiated tumours. Part I. Br J Radiol 1980; 53: 210-27. doi: https://doi. org/10.1259/0007-1285-53-627-210

18. Wyatt RM, Beddoe AH, Dale RG. The effects of delays in radiotherapy treatment on tumour control. Phys Med Biol 2003; 48: 139-55. doi: https://doi.org/10.1088/00319155/48/2/301

19. Del Monte U. Does the cell number 10(9) still really fit one gram of tumor tissue? Cell Cycle 2009; 8: 505-6. doi: https://doi.org/10. 4161/cc.8.3.7608

20. Jones B, Dale RG. The evolution of practical radiobiological modelling. Br J Radiol 2018; 20180097. doi: https://doi.org/10.1259/bjr. 20180097

21. Dale RG. Radiation repair models for clinical application. Br J Radiol 2018; 25: 20180070. doi: https://doi.org/10.1259/bjr.20180070

14. Tan LT, Jones B, Gee A, Kingston RE. An audit of the treatment of carcinoma of the uterine cervix using external beam radiotherapy and a single line source brachytherapy technique. Br J Radiol 1997; 70: 1259-69. doi: https://doi.org/10.1259/bjr. 70.840 .9505845

15. Leroi AM. The lagoon: how Aristotle invented science. Bloomsbury 2014;: 276-8.

22. Friberg S, Mattson S. On the growth rates of human malignant tumors: implications for medical decision making. J Surg Oncol 1997; 65: 284-97. doi: https://doi.org/10.1002/( SICI) 1096-9098(199708)65:4<284::AIDJSO11>3.0.CO;2-2

23. Eaton DJ, Byrne JP. Cosgrove VP Thomas SJ. Unintended doses in radiotherapy-over, under and outside? Brit J radiology 2018; 91 : 20170863.

24. Tomé WA, Fowler JF. On cold spots in tumor subvolumes. Med Phys 2002; 29: 1590-8. doi: https://doi.org/10.1118/1.1485060

25. Borrás C, Barés JP, Rudder D, Amer A, Millán F, Abuchaibe O. Clinical effects in a cohort of cancer patients overexposed during external beam pelvic radiotherapy. In $J$ Radiat Oncol Biol Phys 2004; 59: 538-50. doi: https://doi.org/10.1016/j.ijrobp.2003.10.024

26. Jones B. Errors of Bragg Peak Positioning and their Radiobiological Correction, Chapter 11 in in 'Practical Radiobiology for Proton Therapy planning'. Bristol \& Philadelphia: Institute of Physics Publishing; 2017.

27. Bentzen SM, Constine LS, Deasy JO, Eisbruch A, Jackson A, Marks LB, et al. Quantitative analyses of normal tissue effects in the clinic (QUANTEC): an introduction to the scientific issues. Int $J$ Radiat Oncol Biol Phys 2010; 76(3 Suppl): S3-9PMID. doi: https://doi.org/10.1016/j.ijrobp.2009.09.040
28. Royal College of Radiologists UK. Timely delivery of radical radiotherapy: guidelines for the management of unscheduled treatment interruptions. 4th edition: Publication BFCO (19); 2019.

29. Martell K, Mackenzie J, Kerney W, Lau HY. Management delays in patients with squamous cell cancer of neck node(s) and unknown primary site: a retrospective cohort study. J Otolaryngol Head Neck Surg 2017; 46: 39. doi: https://doi.org/10.1186/s40463-0170217-z

30. Allison P, Franco E, Black M, Feine J. The role of professional diagnostic delays in the prognosis of upper aerodigestive tract carcinoma. Oral Oncol 1998; 34: 147-53. doi: https://doi.org/10.1016/S1368-8375(97) 00088-2

31. Teppo H, Koivunen P, Hyrynkangas K, Alho O-P. Diagnostic delays in laryngeal carcinoma: professional diagnostic delay is a strong independent predictor of survival. Head Neck 2003; 25: 389-94. doi: https://doi. org/10.1002/hed.10208

32. Seoane J, Takkouche B, Varela-Centelles P, Tomás I, Seoane-Romero JM. Impact of delay in diagnosis on survival to head and neck carcinomas: a systematic review with meta-analysis. Clin Otolaryngol 2012; 37: 99-106. doi: https://doi.org/10.1111/j.17494486.2012.02464.x

33. Diaconescu R, Lafond C, Whittom R. Treatment delays in non-small cell lung cancer and their prognostic implications. $J$ Thorac Oncol 2011; 6: 1254-9. doi: https:// doi.org/10.1097/JTO.0b013e318217b623

\section{APPENDIX A}

Figure A1. Plot of the doubling time $(w)$ at any timepoint, the average doubling time $\left(w_{a v}\right)$ during Gompertzian growth. The equivalent doubling time $\left(w_{e q}\right)$, when used with the standard exponential growth equation, provides the same volume over a specified time as would a Gompertz function.

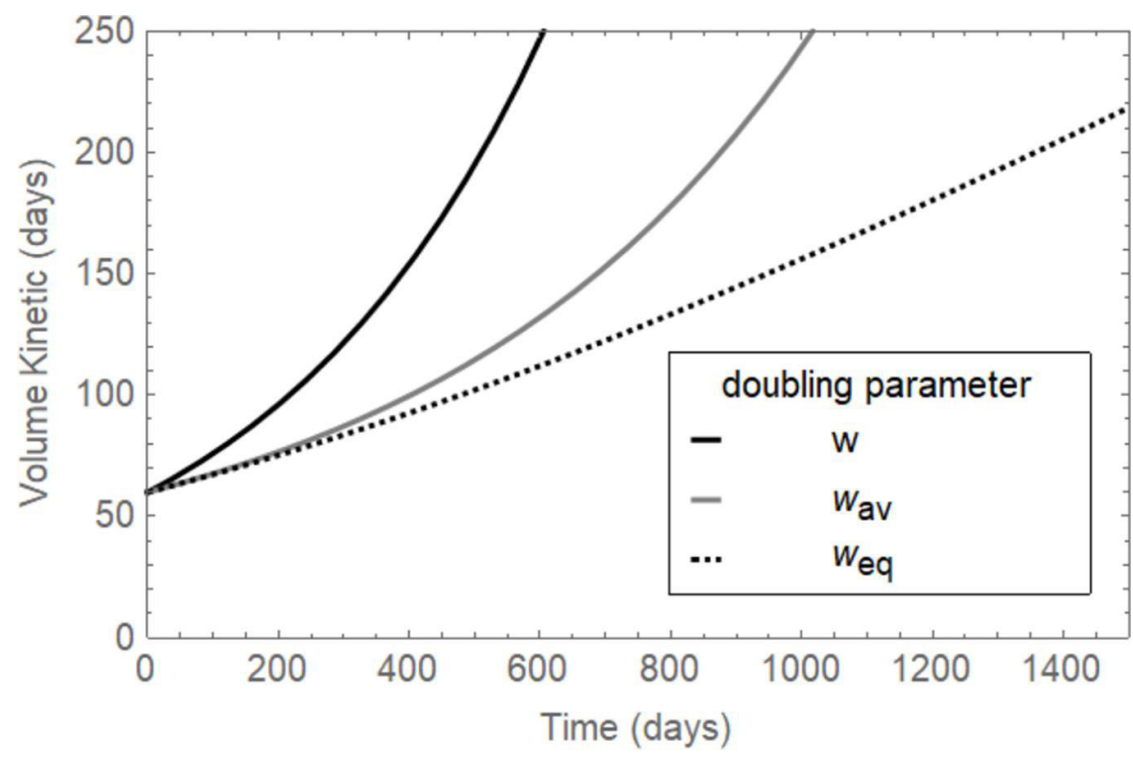




\section{APPENDIX B}

In spherical volumetric studies, where $V_{t}$ and $r_{t}$ are the volume and radius at time $t, V_{0}$ and $r_{0}$ are the original volume and radius, then since for exponential growth with a doubling time $\mathrm{w}$, then

$V_{t}=V_{0} \cdot e^{\frac{0.693 . t}{w}}$

\section{APPENDIX C}

The parameter LE can be further expressed as a mathematical function, as

$$
L E=n\left(\frac{\alpha d}{q_{\alpha}}+\frac{\beta d^{2}}{q_{\beta}}\right)-\frac{0.693 t}{\omega}+\theta
$$

Where $\mathrm{n}$ is the number of radiation treatments each of dose $\mathrm{d}, \alpha$ and $\beta$ are radiosensitivity parameters (which include mitotic and apoptotic cell death), $\mathrm{q}_{\alpha}$ and $\mathrm{q}_{\beta}$ are the factors which reduce radiosensitivity in accordance with the local oxygenation conditions, $t$ is the overall duration of treatment, $\mathrm{w}$ is the average cellular doubling time, and $\theta$ is the total cell kill due to any other factors that are unrelated to treatment e.g. immunological cell death, natural cell loss, effect of chemotherapy etc. which are assumed not to vary for two identical treatments at different times for tumors that are in their early growth phase, including subclinical volumes of tumor.
In terms of the radii, this can be replaced as:

$\frac{4}{3} \pi r_{t}^{3}=\frac{4}{3} \pi r_{0}^{3} e^{\frac{0.693 . t}{w}}$, which leads to $\frac{r_{t}^{3}}{r_{0}^{3}}=e^{\frac{0.693 . t}{w}}$, and $\frac{r_{t}}{r_{0}}=\sqrt[3]{e^{\frac{0.693 . t}{w}}}$, so that $r_{t}=r_{0} e^{\frac{0.693 . t}{3 w}}$, and so the radial doubling time is three times larger than the volume doubling time.

From the Poissonian section equations in the text,

$$
L E=-\log [-\log [\text { assumed } T C P] / n]
$$

The cure probability at the time when treatment was given is $\mathrm{TCP}_{\mathrm{A}}=\operatorname{Exp}[-\mathrm{n} \operatorname{Exp}[-\mathrm{LE}]]$

The cure probability at an earlier time $\tau$, with average cell doubling time $\mathrm{w}$, is:

$$
T C P_{B}=\operatorname{Exp}[-n /(\operatorname{Exp}[0.693 / w]) \operatorname{Exp}[-L E]]
$$

By assuming different TCP values in the expected range, computations will contain the key parameters that determine outcomes and the effect of increasing the treatment delay time $\tau$ can be estimated.

Figure C1. Tumour Control Probability dose response curves for seven different simulated tumours of different radiosensitivities (grey curves) with the averaged population value for all seven tumour shown as the black line which is shallower.

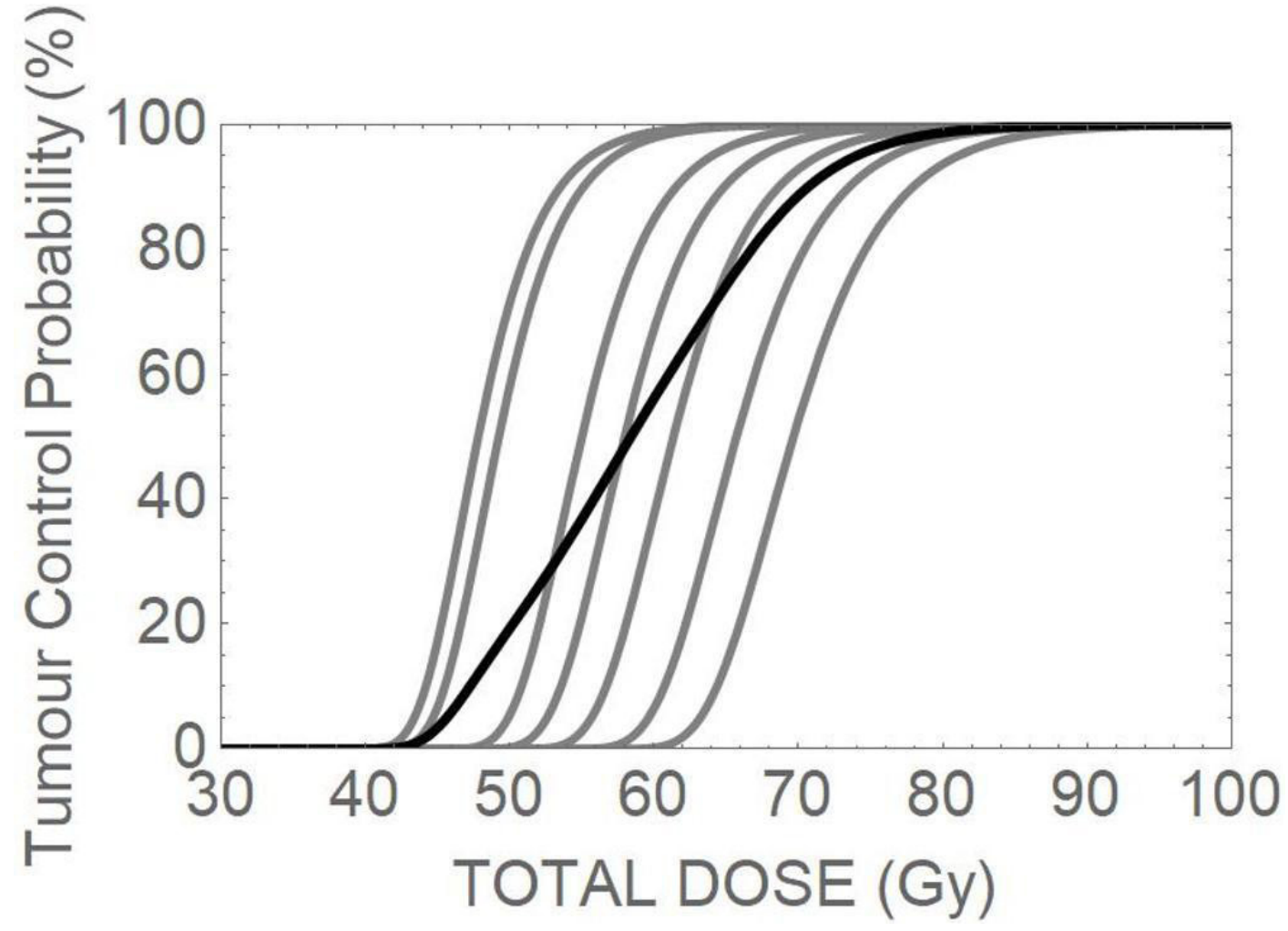

